\title{
On Sums of Dilates
}

\author{
JAVIER CILLERUELO ${ }^{1 \dagger}$, YAHYA O. HAMIDOUNE ${ }^{2}$ \\ and ORIOL SERRA ${ }^{3}$ \\ ${ }^{1}$ Instituto de Ciencias Matemáticas (CSIC-UAM-UC3M-UCM) and Departamento de Matemáticas, \\ Universidad Autónoma de Madrid, 28049-Madrid, Spain \\ (e-mail: franciscojavier.cilleruelo@uam.es)
}

${ }^{2}$ UPMC Université Paris 06, E. Combinatoire, Case 189, 4 Place Jussieu, 75005 Paris, France

(e-mail: hamidoune@math.jussieu.fr)

${ }^{3}$ Universitat Politécnica de Catalunya, Jordi Girona, 1, E-08034 Barcelona, Spain

(e-mail: oserra@ma4.upc.edu)

Received 27 February 2009; revised 26 May 2009

For $k$ prime and $A$ a finite set of integers with $|A| \geqslant 3(k-1)^{2}(k-1)$ ! we prove that $|A+k \cdot A| \geqslant$ $(k+1)|A|-\lceil k(k+2) / 4\rceil$ where $k \cdot A=\{k a: a \in A\}$. We also describe the sets for which equality holds.

\section{Introduction}

Let $k$ be a positive integer and let $A \subset \mathbb{Z}$. We let $k \cdot A=\{k a: a \in A\}$ denote the $k$-dilation of $A$, and let $k A=A+\cdots+A$ ( $k$-times) be the $k$-fold sumset of $A$. We observe that $A+k \cdot A \subset A+$ $k A=(k+1) A$ and that, in general, $A+k \cdot A$ is much smaller than $(k+1) A$. It is well known that $|(k+1) A| \geqslant(k+1)|A|-k$, and that equality holds only if $A$ is an arithmetic progression. Indeed, if $A$ is an arithmetic progression with $|A| \geqslant k$, one can check that $A+k \cdot A=(k+1) A$. So it is a natural problem to study lower bounds for $|A+k \cdot A|$ as well as the description of the extremal cases.

The case $k=1$ is trivial since $|A+A| \geqslant 2|A|-1$ and equality holds for arithmetic progressions. The case $k=2$ (see [3]) is also easy since we can split $A=A_{1} \cup A_{2}$ into the two classes $(\bmod 2)$, and then $|A+2 \cdot A|=\left|A_{1}+2 \cdot A\right|+\left|A_{2}+2 \cdot A\right| \geqslant\left|A_{1}\right|+|2 \cdot A|-1+$ $\left|A_{2}\right|+|2 \cdot A|-1=3|A|-2$. (If $A$ contains only one class we write $A=2 \cdot A^{\prime}+i$ and then

\footnotetext{
$\dagger$ Supported by Project MTM2008-03880 from MYCIT (Spain) and the joint Madrid Region-UAM project TENU3 (CCG08-UAM/ESP-3906).
} 
$|A+2 \cdot A|=\left|A^{\prime}+2 \cdot A^{\prime}\right|$.) It is shown in [2] that $|A+2 \cdot A|=3|A|-2$ only when $A$ is an arithmetic progression.

The cases $k \geqslant 3$ are much more involved. Nathanson [3] proved that $|A+k \cdot A| \geqslant\left\lfloor\frac{7}{2}|A|-\frac{5}{2}\right\rfloor$ for $k \geqslant 3$ and Bukh [1] proved that $|A+3 \cdot A| \geqslant 4|A|-C$ for some constant $C$. Cilleruelo, Silva and Vinuesa [2] obtained the sharp bound and the description of the extremal cases for $k=3$.

Theorem 1.1 ([2]). For any set of integers $A$ we have $|A+3 \cdot A| \geqslant 4|A|-4$. Furthermore, if $|A+3 \cdot A|=4|A|-4$, then $A=3 \cdot\{0, \ldots, n\}+\{0,1\}$, or $A=\{0,1,3\}$, or $A=\{0,1,4\}$ or $A$ is an affine transformation of any of these sets.

They proposed the following conjecture.

Conjecture (Cilleruelo, Silva and Vinuesa [2]). For every positive integer $k$ and a finite set of integers $A$ with sufficiently large cardinality, we have

$$
|A+k \cdot A| \geqslant(k+1)|A|-\lceil k(k+2) / 4\rceil .
$$

Bukh's main theorem [1] states that, for $\left(\lambda_{1}, \ldots, \lambda_{t}\right) \in \mathbb{Z}^{t}$ with $\operatorname{gcd}\left(\lambda_{1}, \ldots, \lambda_{t}\right)=1$,

$$
\left|\lambda_{1} \cdot A+\cdots+\lambda_{t} \cdot A\right| \geqslant\left(\left|\lambda_{1}\right|+\cdots+\left|\lambda_{t}\right|\right)|A|-o(|A|) .
$$

This general result implies $|A+k \cdot A| \geqslant(k+1)|A|-o(|A|)$ in our problem. The existence of a simple proof for $|A+k \cdot A| \geqslant(k+1)|A|-C_{k}$ for $k \geqslant 4$ is implicitly asked by Bukh in [1].

When $k$ is prime we give a positive answer to the above questions by proving a precise version of the conjecture above. In addition we characterize the extremal sets $A$ for the lower bound in that conjecture. Since $|A+k \cdot A|$ is invariant under affine transformations, we will assume without loss of generality that $0 \in A$ and $\operatorname{gcd}(A)=1$.

Theorem 1.2. Let $k$ be a prime and let $A$ be a subset of $\mathbb{Z}$ with $\min A=0, \operatorname{gcd}(A)=1$ and $|A| \geqslant 3|\hat{A}|^{2}(k-1)$ !, where $\hat{A}$ is the projection of $A$ in $\mathbb{Z} / k \mathbb{Z}$. Then

$$
|A+k \cdot A| \geqslant(k+1)|A|-|\hat{A}|(k+1-|\hat{A}|) .
$$

Furthermore, if $|\hat{A}|<k$, equality holds in (1.1) only if

$$
A=k \cdot\{0,1, \ldots, n\}+\{0,1, \ldots,|\hat{A}|-1\}
$$

for some $n$, while if $|\hat{A}|=k$, equality holds in (1.1) only if $A$ is an arithmetic progression.

If $|\hat{A}|=k$, one can obtain

$$
|A+k \cdot A| \geqslant(k+1)|A|-k,
$$

under the weaker hypothesis $|A|>k$, and equality holds only when $A$ is an arithmetic progression. This case is contained in Corollary 2.2 below.

The next corollary follows from Theorem 1.2 and Corollary 2.2. 
Corollary 1.3. Let $k$ be an odd prime and let $A$ be a subset of $\mathbb{Z}$ with $|A| \geqslant 3(k-1)^{2}(k-1)$ !. Then

$$
|A+k \cdot A| \geqslant(k+1)|A|-\lceil k(k+2) / 4\rceil .
$$

Moreover, up to affine transformations, equality holds in (1.3) only if

$$
A=k \cdot\{0,1, \ldots, n\}+\{0,1, \ldots,(k-1) / 2\}
$$

for some $n$.

Theorem 1.2 implies in particular that, for $k$ prime and any set $A$, we have $|A+k \cdot A| \geqslant$ $(k+1)|A|-C_{k}$ for a suitable constant $C_{k}$. Indeed, Lemma 4.1 below shows that inequality holds with $C_{k}=3(k-1)$ !.

Small sets are more difficult to deal with. For example, if $k=3$, Theorem 1.2 covers Theorem 1.1 only when $|A| \geqslant 24$. Smaller sets have to be analysed more carefully, as was done in [2] with a particular approach. Actually the lower bound (1.1) does not hold for an arbitrary set. In [2] it is shown that there exist small sets $A$ for which $|A+k \cdot A| \leqslant(k+1)|A|-P(k)$, where $P$ is a cubic polynomial.

The paper is organized as follows. We first give some notation and preliminary results in Section 2. We then show in Section 3 that, for the class of so-called $k$-full sets, which actually contain the extremal ones, Theorem 1.2 is relatively easy to prove. In Section 4 we give a universal weaker lower bound for the cardinality of $A+k \cdot A$, and we use it to show in the final section that, for sufficiently large sets which are not $k$-full, we get a better lower bound for $|A+k \cdot A|$ than that of Theorem 1.2, thus completing its proof.

\section{Notation and preliminary results}

For two finite non-empty sets of integers $A$ and $B$, it is well known that $|A+B| \geqslant|A|+|B|-1$, and that equality holds only if either $\min \{|A|,|B|\}=1$ or both $A$ and $B$ are arithmetic progressions with the same common difference. We next give a generalization of the above inequality for $|A+k \cdot B|$.

A maximal subset of $X \subset \mathbb{Z}$ of congruent elements modulo $k$ will be called a $k$-component of $X$.

Lemma 2.1. For arbitrary non-empty sets of integers $A$ and $B$ with $|B|>1$, we have

$$
|A+k \cdot B| \geqslant|A|+|\hat{A}|(|B|-1),
$$

where $\hat{A}$ denotes the natural projection of $A$ onto $\mathbb{Z} / k \mathbb{Z}$.

Furthermore, if equality holds and $A$ has a k-component $C$ with $|C|>1$, then both $C$ and $k \cdot B$ are arithmetic progressions with the same difference.

Proof. Observe that $A+k \cdot B$ is the disjoint union $\cup_{i \in \hat{A}}\left(A_{i}+k \cdot B\right)$, where $A_{i}$ are the distinct $k$-components of $A$. Write $A_{i}=k \cdot X_{i}+u_{i}$. We have

$$
\begin{aligned}
|A+k \cdot B| & =\left|\cup_{i \in \hat{A}}\left(k \cdot X_{i}+u_{i}+k \cdot B\right)\right| \\
& =\sum_{i \in \hat{A}}\left|X_{i}+B\right| \geqslant \sum_{i \in \hat{A}}\left(\left|X_{i}\right|+|B|-1\right)=|A|+|\hat{A}|(|B|-1) .
\end{aligned}
$$


To prove the second part of the statement, suppose that equality holds and let $C=A_{r}=k$. $X_{r}+u_{r}$. Then $\left|X_{r}+B\right|=\left|X_{r}\right|+|B|-1$, which implies that both $X_{r}$ and $B$ are arithmetic progressions with the same difference and the same is true of $A_{r}$ and $k \cdot B$.

Lemma 2.1 easily handles the case when $|\hat{A}|=k$, as described in the next corollary.

Corollary 2.2. Let $A$ be a set of integers with $|\hat{A}|=k$ and $|A|>k$. Then we have

$$
|A+k \cdot A| \geqslant(k+1)|A|-k,
$$

and equality holds only if $A$ is an arithmetic progression.

Proof. The inequality follows from Lemma 2.1. For the inverse part, we observe that $\left|A_{r}\right| \geqslant 2$ for some $r$, and Lemma 2.1 implies that the set $k \cdot A$ must be an arithmetic progression. Hence $A$ must be an arithmetic progression as well.

Throughout the paper we use the following notation. For a set $A$ we let $\hat{A}$ denote the natural projection of $A$ on $\mathbb{Z} / k \mathbb{Z}$. We simply write $\hat{a}$ if $A=\{a\}$. We write $j=|\hat{A}|$ and $A_{1}, \ldots, A_{j}$ for the distinct classes of $A$ modulo $k$. We also write $A_{i}=k \cdot X_{i}+u_{i}, i=1, \ldots, j$ for some distinct $u_{i}$ modulo $k$. Thus,

$$
A=\bigcup_{i=1}^{j} A_{i}=\bigcup_{i=1}^{j}\left(k \cdot X_{i}+u_{i}\right) .
$$

Unless explicitly stated, we will always assume that $\left|A_{1}\right| \geqslant\left|A_{2}\right| \geqslant \cdots \geqslant\left|A_{j}\right|$. Also, we write

$$
\begin{array}{ll}
F=\left\{i:\left|\hat{X}_{i}\right|=k\right\}, & A_{F}=\bigcup_{i \in F} A_{i}, \\
E=\left\{i: 0<\left|\hat{X}_{i}\right|<k\right\}, & A_{E}=\bigcup_{i \in E} A_{i} .
\end{array}
$$

Let

$$
\Delta_{r s}=\left(A_{r}+k \cdot A\right) \backslash\left(A_{r}+k \cdot A_{s}\right),
$$

so that

$$
\left|A_{r}+k \cdot A\right|=\left|A_{r}+k \cdot A_{s}\right|+\left|\Delta_{r s}\right|=\left|X_{r}+k \cdot X_{s}\right|+\left|\Delta_{r s}\right| .
$$

Lemma 2.3. For each subset $I \subset\{1,2, \ldots, j\}$ and each $r \in\{1,2, \ldots, j\}$, we have:

(i) $\sum_{i \in I}\left|\Delta_{i i}\right| \geqslant|I|(|I|-1)$,

(ii) $\sum_{i \in I}\left|\Delta_{r i}\right| \geqslant|I|(|I|-1)$,

Proof. Let

$$
\Delta_{r s}^{+}=\left(A_{r}+k \cdot A\right) \backslash\left(-\infty, \max \left(A_{r}+k \cdot A_{s}\right)\right],
$$

and

$$
\Delta_{r s}^{-}=\left(A_{r}+k \cdot A\right) \backslash\left[\min \left(A_{r}+k \cdot A_{s}\right), \infty\right),
$$


so that

$$
\left|\Delta_{r s}\right| \geqslant\left|\Delta_{r s}^{+}\right|+\left|\Delta_{r s}^{-}\right| .
$$

Let $\Gamma^{+}(s)=\left\{h: \max \left(A_{s}\right)<\max \left(A_{h}\right)\right\} \quad$ and $\quad \Gamma^{-}(s)=\left\{h: \min \left(A_{s}\right)>\min \left(A_{h}\right)\right\}$. Clearly $\max \left(A_{r}+k \cdot A_{s}\right)<\max \left(A_{r}+k \cdot A_{h}\right)$, for every $h \in \Gamma^{+}(s)$. Since, for distinct $h$, the elements on the right-hand side of the last inequality belong to distinct congruence classes modulo $k^{2}$, we have $\left|\Delta_{r s}^{+}\right| \geqslant\left|\Gamma^{+}(s)\right|$. By replacing $A$ by $-A$, we obtain $\left|\Delta_{r s}^{-}\right| \geqslant\left|\Gamma^{-}(s)\right|$.

Observe that $\left|\Gamma^{+}(u)\right|>\left|\Gamma^{+}(v)\right|$ if $\max \left(A_{u}\right)<\max \left(A_{v}\right)$. In particular, the numbers $\left|\Gamma^{+}(u)\right|$, $u=1, \ldots, j$ are pairwise distinct. Since $\left|\Gamma^{+}(u)\right| \leqslant j-1$, it follows that

$$
\left\{\left|\Gamma^{+}(u)\right|: u=1,2, \ldots, j\right\}=\{0,1, \ldots, j-1\} .
$$

By replacing $A$ by $-A$, we get $\left\{\left|\Gamma^{-}(u)\right|: u=1,2, \ldots, j\right\}=\{0,1, \ldots, j-1\}$ as well. Therefore,

$$
\sum_{i \in I}\left|\Delta_{i i}\right| \geqslant \sum_{i \in I}\left|\Delta_{i i}^{+}\right|+\sum_{i \in I}\left|\Delta_{i i}^{-}\right| \geqslant \sum_{i \in I}\left|\Gamma^{+}(i)\right|+\sum_{i \in I}\left|\Gamma^{-}(i)\right| \geqslant|I|(|I|-1),
$$

which proves (i). Similarly,

$$
\sum_{i \in I}\left|\Delta_{r i}\right| \geqslant \sum_{i \in I}\left|\Gamma^{+}(i)\right|+\sum_{i \in I}\left|\Gamma^{-}(i)\right| \geqslant|I|(|I|-1),
$$

and (ii) follows.

Lemma 2.4. Let $k$ be a prime and assume the notation above. Then:

(i) if $i \in E$ then $\left|\Delta_{i i}\right| \geqslant\left|A_{s}\right|$ for any $s \neq i$,

(ii) $\sum_{i \in E}\left|\Delta_{i i}\right| \geqslant(|E|-1)\left|A_{1}\right|+\left|A_{2}\right|$.

Proof. (i) Suppose that $\hat{X}_{i}+x=\hat{X}_{i}+y$ for distinct $x, y \in \mathbb{Z} / k \mathbb{Z}$. Then $\hat{X}_{i}=\hat{X}_{i}+(y-x)=$ $\hat{X}_{i}+2(y-x)=\cdots=\hat{X}_{i}+(k-1)(y-x)$, which implies $\hat{X}_{i}=\mathbb{Z} / k \mathbb{Z}$, as $k$ is prime. Hence, if $i \in E$ and $s \neq i$ then $\hat{X}_{i}+\hat{u}_{s} \neq \hat{X}_{i}+\hat{u}_{i}$. Thus $\left|\left(\hat{X}_{i}+\hat{u}_{s}\right) \backslash\left(\hat{X}_{i}+\hat{u}_{i}\right)\right| \geqslant 1$. Now we have

$$
\begin{aligned}
\left|\Delta_{i i}\right| & =\left|\left(A_{i}+k \cdot A\right) \backslash\left(A_{i}+k \cdot A_{i}\right)\right| \geqslant\left|\left(k \cdot X_{i}+u_{i}+k \cdot A_{s}\right) \backslash\left(k \cdot X_{i}+u_{i}+k \cdot A_{i}\right)\right| \\
& =\left|\left(X_{i}+A_{s}\right) \backslash\left(X_{i}+A_{i}\right)\right|=\left|\left(X_{i}+k \cdot X_{s}+u_{s}\right) \backslash\left(X_{i}+k \cdot X_{i}+u_{i}\right)\right| \\
& \geqslant\left|X_{s}\right|\left|\left(\hat{X}_{i}+\hat{u}_{s}\right) \backslash\left(\hat{X}_{i}+\hat{u}_{i}\right)\right| \geqslant\left|X_{s}\right|=\left|A_{s}\right| .
\end{aligned}
$$

(ii) We observe that (i) implies that $\left|\Delta_{i i}\right| \geqslant\left|A_{1}\right|$ for all $i \in E$ except for $i=1$, when $1 \in E$. In that case we have $\left|\Delta_{11}\right| \geqslant\left|A_{2}\right|$.

\section{Full sets}

We say that a set $A$ is $k$-full if $\left|\hat{X}_{i}\right|=k$ for each $i=1,2, \ldots, j$. The following lemma proves Theorem 1.2 for $k$-full sets and all $k$ with a weaker condition on their cardinality. Since Corollary 2.2 proves Theorem 1.2 for $j=k$, we can assume that $j<k$.

Lemma 3.1. Let $A$ be a finite $k$-full set of integers with $\min (A)=0, \operatorname{gcd}(A)=1,|A|>j k$ and $j<k$. Then

$$
|A+k \cdot A| \geqslant(k+1)|A|-j(k-j+1)
$$


Moreover, equality holds if and only if

$$
A=k \cdot\{0,1, \ldots, n\}+\{0,1, \ldots, j-1\}
$$

for some $n$.

Proof. We apply (2.5) and Lemma 2.1 to get, for each $s=1, \ldots, j$,

$$
\begin{aligned}
|A+k \cdot A| & =\sum_{i=1}^{j}\left|A_{i}+k \cdot A\right|=\sum_{i=1}^{j}\left(\left|X_{i}+k \cdot X_{s}\right|+\left|\Delta_{i s}\right|\right) \\
& \geqslant \sum_{i=1}^{j}\left(\left|X_{i}\right|+k\left(\left|X_{s}\right|-1\right)+\left|\Delta_{i s}\right|\right) \geqslant|A|+k j\left|X_{s}\right|-k j+\sum_{i=1}^{j}\left|\Delta_{i s}\right| .
\end{aligned}
$$

If we sum over all $s=1, \ldots, j$ and divide by $j$, we obtain

$$
\begin{aligned}
|A+k \cdot A| & \geqslant(k+1)|A|-k j+\frac{1}{j} \sum_{s=1}^{j} \sum_{i=1}^{j}\left|\Delta_{i s}\right| \\
& =(k+1)|A|-k j+\frac{1}{j} \sum_{i=1}^{j} \sum_{s=1}^{j}\left|\Delta_{i s}\right| \\
& \geqslant(k+1)|A|-j(k+1-j),
\end{aligned}
$$

due to Lemma 2.3(ii). This proves the lower bound.

For the inverse part of the lemma and only until the end of this proof, we next order the $k$-components $A_{1}, A_{2}, \ldots, A_{j}$ of $A$ in such a way that $0=m_{1}<m_{2}<\cdots<m_{j}$, where $m_{i}=$ $\min \left(A_{i}\right)$ (so we do not assume they are decreasing in cardinality).

Suppose that equality holds in (1.1). Since there is equality in (3.1), we have $\left|X_{i}+k \cdot X_{s}\right|=$ $\left|X_{i}\right|+k\left(\left|X_{S}\right|-1\right)$ for all $i$, s. Since $|A|>j k$, we have $\left|X_{i}\right|>k$ for some $i$, so that one of the $k$-components of this $X_{i}$ has at least two elements. Lemma 2.1 implies that all $X_{s}$ are arithmetic progressions with the same difference $d$. So, for $i=1, \ldots, j$ we have

$$
A_{i}=(k d) \cdot\left\{0,1, \ldots, n_{i}\right\}+m_{i}
$$

for some $n_{i} \geqslant k-1$, where $m_{i}=\min \left(A_{i}\right)$ and $|A|=\sum_{i=1}^{j}\left(n_{i}+1\right)$.

Observe that, since $n_{i} \geqslant k-1$, we have

$$
\begin{aligned}
A_{i}+k \cdot A_{r} & =m_{i}+(k d) \cdot\left\{0,1, \ldots n_{i}\right\}+k \cdot\left(m_{r}+(k d) \cdot\left\{0,1, \ldots, n_{r}\right\}\right) \\
& =m_{i}+k m_{r}+(k d) \cdot\left(\left\{0,1, \ldots, n_{i}\right\}+k \cdot\left\{0,1, \ldots, n_{r}\right\}\right) \\
& =m_{i}+k m_{r}+(k d) \cdot\left\{0,1, \ldots, n_{i}+k n_{r}\right\},
\end{aligned}
$$

so that $A_{i}+k \cdot A_{r}$ is an arithmetic progression for each $i$ and $r$.

First we will prove that $m_{r} \equiv 0(\bmod d)$ for all $r$. Otherwise, if we write $R_{0}$ for those $r$ with $m_{r} \equiv 0(\bmod d)\left(\right.$ which contains $\left.m_{1}\right)$ and $R_{1}$ for those $r$ with $m_{r} \neq \equiv 0(\bmod d)($ which is also 
non-empty by assumption), we have

$$
\begin{aligned}
|A+k \cdot A| & =\sum_{i=1}^{j}\left|A_{i}+k \cdot A\right|=\sum_{i=1}^{j}\left|\bigcup_{r}\left(A_{i}+k \cdot A_{r}\right)\right| \\
& =\sum_{i=1}^{j}\left|\bigcup_{r}\left(m_{i}+k m_{r}+(k d) \cdot\left\{0,1, \ldots, n_{i}+k n_{r}\right\}\right)\right| \\
& =\sum_{i=1}^{j}\left|\bigcup_{r}\left(m_{r}+d \cdot\left\{0,1, \ldots, n_{i}+k n_{r}\right\}\right)\right| \\
& =\sum_{i=1}^{j}\left(\left|\bigcup_{r \in R_{0}}\left(d \cdot\left\{0, \ldots, k n_{r}+n_{i}\right\}+m_{r}\right)\right|\right. \\
& \left.+\left|\bigcup_{r \in R_{1}}\left(d \cdot\left\{0, \ldots, k n_{r}+n_{i}\right\}+m_{r}\right)\right|\right) \\
& \geqslant \sum_{i=1}^{j}\left(n_{i}+1+k \max _{r \in R_{0}} n_{r}\right)+\sum_{i=1}^{j}\left(n_{i}+1+k \max _{r \in R_{1}} n_{r}\right) \\
& =2|A|+k j\left(\max _{r \in R_{0}} n_{r}+\max _{r \in R_{1}} n_{r}\right) \geqslant 2|A|+k j\left(k-1+\max _{r} n_{r}\right) \\
& =2|A|+k j\left(k-2+\max _{r}\left(n_{r}+1\right)\right) \geqslant 2|A|+k j\left(k-2+\frac{|A|}{j}\right) \\
& =2+k)|A|>(k+1)|A|,
\end{aligned}
$$

and equality (1.1) cannot hold.

Now, since $\operatorname{gcd}(A)=1$, we have that $d=1$. It follows, by (3.3), that

$$
A_{i}+k \cdot A=\bigcup_{r=1}^{j}\left(A_{i}+k \cdot A_{r}\right)=\bigcup_{r=1}^{j}\left(m_{i}+k m_{r}+k \cdot\left\{0,1, \ldots, k n_{r}+n_{i}\right\}\right) .
$$

By using the notation from the proof of Lemma 2.3, for each $i$ and for each $r \geqslant 2$, the set $\Delta_{i r}^{-}=\left(A_{i}+k \cdot A\right) \backslash\left[\min \left(A_{i}+k \cdot A_{r}\right), \infty\right)$ clearly contains $m_{i}+k m_{1}, m_{i}+k m_{2}, \ldots, m_{i}+k m_{r-1}$. It follows that

$$
\sum_{r=1}^{j}\left|\Delta_{i r}^{-}\right| \geqslant \sum_{r=2}^{j}(r-1)=j(j-1) / 2 .
$$

By the analogous argument on $-A$ we also have $\sum_{r=1}^{j}\left|\Delta_{i r}^{+}\right| \geqslant j(j-1) / 2$.

Since there is equality in (3.2), we have $\sum_{s=1}^{j} \Delta_{i s}=j(j-1)$ for each $i$. It follows that $\sum_{r=1}^{j}\left|\Delta_{i r}^{-}\right|=\sum_{r=1}^{j}\left|\Delta_{i r}^{+}\right|=j(j-1) / 2$. Hence

$$
\Delta_{i r}^{-}=m_{i}+k \cdot\left\{m_{1}, m_{2}, \ldots, m_{r-1}\right\}, r=2,3, \ldots, j .
$$

We claim that $m_{r-1}+1=m_{r}$ for each $2 \leqslant r \leqslant j$. Suppose, on the contrary, that $m_{r-1}+1<$ $m_{r}$ (we have assumed that $\left.0=m_{1}<\cdots<m_{j}\right)$. Then $m_{i}+k\left(m_{r-1}+1\right)<\min \left(A_{i}+k \cdot A_{r}\right)$. On 
the other hand, by (3.4), we have $m_{i}+k\left(m_{r-1}+1\right) \in m_{i}+k m_{r}+k \cdot\left\{0,1, \ldots, k n_{r}+n_{i}\right\} \subset A_{i}+$ $k \cdot A$. Thus $m_{i}+k\left(m_{r-1}+1\right) \in \Delta_{i r}^{-}$, which is a contradiction because $\max \Delta_{i r}^{-}=m_{i}+k m_{r-1}<$ $m_{i}+k\left(m_{r-1}+1\right)$.

Since $m_{1}=0$, we conclude that $m_{r}=r-1$ for $r=1, \ldots, j$. Thus $[0, j-1] \subset A$.

By applying the above argument to $-A+\max (A)$, we conclude that the set $A$ also contains the interval $[\max (A)-(j-1), \max (A)]$. Since each $A_{i}$ is an arithmetic progression of difference $k$ and $j<k$, we must have $\max (A)-(j-1) \equiv 0(\bmod k)$ and $n_{1}=\cdots=n_{j}$. This completes the proof.

\section{A general lower bound}

In this section we give a weaker lower bound for $|A+k \cdot A|$ valid for every finite set $A$ of integers and $k$ prime.

Lemma 4.1. Let $k$ be a prime and let $A$ be a finite non-empty subset of $\mathbb{Z}$. We have

$$
|A+k \cdot A| \geqslant(k+1)|A|-3(k-1) !
$$

Proof. Let $t$ be the largest integer such that, for every finite set $X$ of integers,

$$
|X+k \cdot X| \geqslant(t+1)|X|-3(t-1) !
$$

Suppose that $t<k$ and let $A$ be a critical set, verifying $|A+k \cdot A|<(t+2)|A|-3 t$ !. Without loss of generality we may assume that $0 \in A_{1}$ and $\operatorname{gcd}(A)=1$. In particular $j=|\hat{A}| \geqslant 2$.

Lemma 2.1 gives $|A+k \cdot A| \geqslant(j+1)|A|-j$. Therefore $t \geqslant j$.

We have

$$
|A+k \cdot A|=\sum_{i \in F}\left|A_{i}+k \cdot A\right|+\sum_{i \in E}\left|A_{i}+k \cdot A\right| .
$$

We have

$$
\begin{aligned}
\sum_{i \in F}\left|A_{i}+k \cdot A\right| & \geqslant \sum_{i \in F}\left|A_{i}+k \cdot A_{1}\right| \\
& =\sum_{i \in F}\left|X_{i}+k \cdot X_{1}\right| \\
(\text { by Lemma 2.1) } & \geqslant \sum_{i \in F}\left(\left|X_{i}\right|+k\left(\left|X_{1}\right|-1\right)\right) \\
& =\sum_{i \in F}\left(\left|A_{i}\right|+k\left(\left|A_{1}\right|-1\right)\right) \\
(\text { since } t \leqslant k-1) & \geqslant\left|A_{F}\right|+(t+1)|F|\left(\left|A_{1}\right|-1\right) \\
\text { (since } \left.t\left|A_{1}\right||F| \geqslant t\left|A_{F}\right|\right) & \geqslant(t+1)\left|A_{F}\right|+|F|\left|A_{1}\right|-(t+1)|F| .
\end{aligned}
$$


On the other hand, by (2.5), Lemma 2.4(ii) and the assumption on $t$,

$$
\begin{aligned}
\sum_{i \in E}\left|A_{i}+k \cdot A\right| & =\sum_{i \in E}\left(\left|A_{i}+k \cdot A_{i}\right|+\left|\Delta_{i i}\right|\right) \\
& \geqslant \sum_{i \in E}\left((t+1)\left|A_{i}\right|-3(t-1) !\right)+\sum_{i \in E}\left|\Delta_{i i}\right| \\
& \geqslant(t+1)\left|A_{E}\right|-3|E|(t-1) !+(|E|-1)\left|A_{1}\right|+\left|A_{2}\right| .
\end{aligned}
$$

By substitution of (4.3) and (4.4) in (4.2), we get

$$
\begin{aligned}
|A+k \cdot A| & \geqslant(t+1)|A|+(|F|+|E|-1)\left|A_{1}\right|+\left|A_{2}\right|-(t+1)|F|-3|E|(t-1) ! \\
& \geqslant(t+2)|A|-(t+1)|F|-3|E|(t-1) !
\end{aligned}
$$

since $(|F|+|E|-1)\left|A_{1}\right|+\left|A_{2}\right|=(j-1)\left|A_{1}\right|+\left|A_{2}\right| \geqslant\left|A_{1}\right|+\left|A_{2}\right|+\cdots+\left|A_{j}\right|=|A|$. Finally, since $|E|+|F|=j \leqslant t$, we have

$$
\begin{aligned}
3|E|(t-1) !+(t+1)|F| & \leqslant 3(t-|F|)(t-1) !+(t+1)|F| \\
& \leqslant 3 t !+|F|(t+1-3(t-1) !) \leqslant 3 t !
\end{aligned}
$$

which contradicts our choice of $A$. This contradiction proves the statement.

\section{Proof of Theorem 1.2}

Suppose now that $|A| \geqslant 3 j^{2}(k-1)$ !. By Lemma 3.1 and Corollary 2.2 we may assume $E \neq \emptyset$ and $j<k$.

Case 1. There exists $s \geqslant 2$ with $s \in E$.

By (2.5), Lemma 4.1 and Lemma 2.4(i), we obtain

$$
\begin{aligned}
|A+k \cdot A| & =\left|A_{s}+k \cdot A\right|+\sum_{i \neq s}\left|A_{i}+k \cdot A\right| \\
& \geqslant\left|X_{s}+k \cdot X_{s}\right|+\left|\Delta_{s S}\right|+\sum_{i \neq s}\left|X_{i}+k \cdot X_{i}\right| \\
& \geqslant(k+1)\left|A_{s}\right|-3(k-1) !+\left|A_{1}\right|+\sum_{i \neq s}\left((k+1)\left|A_{i}\right|-3(k-1) !\right) \\
& \geqslant(k+1)|A|-3 j(k-1) !+\frac{|A|}{j} \\
& \geqslant(k+1)|A|,
\end{aligned}
$$

since $|A| \geqslant 3 j^{2}(k-1)$ !.

Case 2. $E=\{1\}$.

In this case, since $\left|\hat{X}_{2}\right|=k$, Lemma 2.1 implies that

$$
\left|X_{2}+k \cdot X_{1}\right| \geqslant\left|X_{2}\right|+k\left(\left|X_{1}\right|-1\right)=\left|A_{2}\right|+k\left|A_{1}\right|-k .
$$

We observe also (by Lemma 2.4(i)) that $\left|\Delta_{11}\right| \geqslant\left|A_{2}\right|$. 
Then, by (2.5), Lemma 4.1, Lemma 2.4(i) and (5.1), we have

$$
\begin{aligned}
|A+k \cdot A|=\left|A_{1}+k \cdot A\right|+\left|A_{2}+k \cdot A\right|+\sum_{i \geqslant 3}\left|A_{i}+k \cdot A\right| \\
\geqslant\left|X_{1}+k \cdot X_{1}\right|+\left|\Delta_{11}\right|+\left|X_{2}+k \cdot X_{1}\right|+\sum_{3 \leqslant i \leqslant j}\left|X_{i}+k \cdot X_{i}\right| \\
\geqslant(k+1)\left|A_{1}\right|-3(k-1) !+\left|A_{2}\right|+\left(\left|A_{2}\right|+k\left|A_{1}\right|-k\right) \\
\quad \quad+\sum_{3 \leqslant i \leqslant j}\left((k+1)\left|A_{i}\right|-3(k-1) !\right) \\
\geqslant \\
\geqslant\left|A_{1}\right|+(k+1)|A|-3(j-1)(k-1) !-k \\
\geqslant \frac{|A|}{j}+(k+1)|A|-3 j(k-1) ! \\
\geqslant(k+1)|A|,
\end{aligned}
$$

since $|A| \geqslant 3 j^{2}(k-1)$ !.

This completes the proof.

\section{Acknowledgements}

The authors wish to thank an anonymous referee for very helpful remarks, and particularly for the suggested simplification of the proof of Lemma 3.1.

\section{References}

[1] Bukh, B. (2008) Sums of dilates. Combin. Probab. Comput. 17 627-639.

[2] Cilleruelo, J., Silva, M. and Vinuesa, C. A sumset problem. Preprint.

[3] Nathanson, M. B. Inverse problems for linear forms over finite sets of integers. Available from: arXiv: 0708.2304. 